\title{
Inconsistencies in authoritative national paediatric workforce data sources
}

\author{
Amy R. Allen ${ }^{1}$ BBNSc, BPsySci(Hons), GradDipAOD, Research Assistant \\ Richard Doherty ${ }^{2}$ MBBS, FRACP, Dean \\ Andrew M. Hilton ${ }^{2}$ BSc, Project Officer \\ Gary L. Freed ${ }^{1,3} \mathrm{MD}, \mathrm{MPH}$, Director \\ ${ }^{1}$ Centre for Health Policy, Melbourne School of Population and Global Health, The University of Melbourne, \\ 207 Bouverie Street, Carlton, Vic. 3053, Australia. Email: amy.allen@unimelb.edu.au \\ ${ }^{2}$ The Royal Australasian College of Physicians (RACP), Level 2, 417 St Kilda Road, Melbourne, Vic. 3004, Australia. \\ Email: richard.doherty@racp.edu.au; andrew.hilton@racp.edu.au \\ ${ }^{3}$ Corresponding author. Email: Gary.freed@unimelb.edu.au
}

\begin{abstract}
Objective. National health workforce data are used in workforce projections, policy and planning. If data to measure the current effective clinical medical workforce are not consistent, accurate and reliable, policy options pursued may not be aligned with Australia's actual needs. The aim of the present study was to identify any inconsistencies and contradictions in the numerical count of paediatric specialists in Australia, and discuss issues related to the accuracy of collection and analysis of medical workforce data.

Methods. This study compared respected national data sources regarding the number of medical practitioners in eight fields of paediatric speciality medical (non-surgical) practice. It also counted the number of doctors listed on the websites of speciality paediatric hospitals and clinics as practicing in these eight fields.

Results. Counts of medical practitioners varied markedly for all specialties across the data sources examined. In some fields examined, the range of variability across data sources exceeded $450 \%$.

Conclusions. The national datasets currently available from federal and speciality sources do not provide consistent or reliable counts of the number of medical practitioners. The lack of an adequate baseline for the workforce prevents accurate predictions of future needs to provide the best possible care of children in Australia.

What is known about the topic? Various national data sources contain counts of the number of medical practitioners in Australia. These data are used in health workforce projections, policy and planning.

What does this paper add? The present study found that the current data sources do not provide consistent or reliable counts of the number of practitioners in eight selected fields of paediatric speciality practice. There are several potential issues in the way workforce data are collected or analysed that cause the variation between sources to occur.

What are the implications for practitioners? Without accurate data on which to base decision making, policy options may not be aligned with the actual needs of children with various medical needs, in various geographic areas or the nation as a whole.
\end{abstract}

Additional keywords: data accuracy, health policy, medical specialist.

Received 27 April 2016, accepted 13 September 2016, published online 28 October 2016

\section{Introduction}

Many medical problems necessitate treatment by a medical practitioner ('doctor') with advanced education and training in a specific area of medicine. Ensuring an adequate supply of doctors in specific specialties is an essential function of the healthcare system. Fundamental to ensuring an adequate supply and addressing any perceived shortages is knowledge of the current number of doctors in the workforce. Government agencies or professional societies cannot determine how many additional doctors may be needed in a given speciality to adequately address the health needs of the population unless they know many are currently in practice.

A 'medical specialist' is defined as a practitioner who holds registration under the Australian Health Practitioner Regulation 
National Law in a recognised speciality. ${ }^{1}$ The Australian Health Workforce Ministerial Council is responsible for developing a list of medical specialties. ${ }^{1}$ Within each speciality there may be multiple specific fields of practice. To register as a medical specialist, a doctor must meet specific eligibility, suitability and qualification requirements. ${ }^{2}$ For some specialties, the training process can take up to 15 years. ${ }^{3}$ Provided they meet all relevant criteria, medical practitioners may register in one or more specialties or fields of practice. 'Paediatrics and child health' ('paediatrics') is a medical speciality within which there are 21 distinct fields of practice, such as paediatric cardiology and paediatric neurology. ${ }^{4}$

The Medical Board of Australia (MBA) is responsible for the regulation and registration of all practicing doctors. The actual registration process is administered by the Australian Health Practitioner Regulation Agency (AHPRA). Registration data from the MBA indicates that, as of September 2015, Australia had 63100 registered medical specialists, with 2477 of these being registered to practice in paediatrics. ${ }^{5}$

Significant federal and state government effort is directed towards maintaining an adequate medical specialist workforce now and into the future. ${ }^{6-8}$ Discussion regarding medical specialist workforce supply and planning occurs in multiple arenas, including government reports and policy, the media and academic literature. ${ }^{3,6,9}$ Concerns regarding current and future shortages of medical specialists are particularly marked for regional and rural areas. ${ }^{10,11}$ A large federal government-commissioned study published in 2012 identified specific workforce issues, including maldistribution of doctors across the medical specialties, and a projected undersupply in many specialties and fields of practice. ${ }^{3}$ However, assessment of the accuracy of the data from which the baseline assumptions of this report were developed did not take place. ${ }^{3,9}$

It is crucial to have consistent and accurate data in order to attain a true picture of the effective clinical workforce. Several data sources are available that contain numerical counts of the number of medical specialists in Australia ('head counts'). These data are derived from a variety of resources, and organisations often use different methods to collect, process and analyse data.

Research in other countries has identified significant variation in workforce numerical counts between different data sources. ${ }^{12,13}$ Such variation significantly affects the face validity of any workforce analysis and interpretation and, in turn, impacts on the effectiveness of workforce policy and planning decisions. ${ }^{12}$

The present study compared paediatric specialist workforce data from four existing respected national data sources, as well as counts obtained from speciality paediatric hospital and clinic websites, of the number of doctors working in eight representative fields of paediatric speciality practice. The aim was to identify any inconsistencies and contradictions in the numerical count of paediatric specialists in Australia and discuss issues related to the accuracy of collection and analysis of medical workforce data.

\section{Methods}

The present study compared respected national data sources regarding the number of medical practitioners identified or listed in eight fields of paediatric speciality medical (non-surgical) practice. The fields of speciality practice included in the study were arbitrarily determined with the goal of being representative of all paediatric speciality fields and were cardiology, endocrinology, gastroenterology and hepatology, immunology and allergy, nephrology, neurology, respiratory and sleep medicine, and rheumatology. The present study received ethics approval from the University of Melbourne Human Research Ethics Committee.

The national datasets included in the study were as follows: (1) the 2013 National Health Workforce Dataset (NHWD); ${ }^{14,15}$ (2) the 2013 AHPRA workforce survey; ${ }^{16}$ (3) the 2014 Royal Australian College of Physicians (RACP) member database; and (4) $2015 \mathrm{MBA}$ registrant data. ${ }^{5}$ The datasets contained only numeric counts, which precluded the individual identification of specific doctors. Specific details regarding the datasets are given in Table 1.

The AHPRA workforce survey asks doctors to report up to two speciality fields in which they worked the most hours during the preceding week. The speciality field in which they report working the most hours is designated by AHPRA to be their primary speciality and, if they report hours worked in another speciality field, that is designated as their secondary field of practice. The choices include both adult and paediatric speciality designations. For paediatric specialties, specialties

Table 1. Dataset details

NHWD, national health workforce dataset; ${ }^{14}$ AHPRA, Australian Health Practitioner Regulation Agency; RACP, Royal Australian College of Physicians; MBA, Medical Board of Australia ${ }^{5}$

\begin{tabular}{|c|c|c|}
\hline Dataset & Year & Details \\
\hline NHWD & 2013 & $\begin{array}{l}\text { AHPRA registration data integrated with AHPRA workforce survey data } \\
\text { Registration data: compulsory } \\
\text { Survey data: voluntary }(88.6 \% \text { response rate) } \\
\text { Completed annually during registration renewal }\end{array}$ \\
\hline AHPRA workforce survey & 2013 & $\begin{array}{l}\text { AHPRA workforce survey } \\
\text { Voluntary }(88.6 \% \text { response rate) } \\
\text { Completed annually during registration renewal }\end{array}$ \\
\hline RACP member database & 2014 & $\begin{array}{l}\text { Specialists currently registered as Fellows of the College } \\
\text { Voluntary }\end{array}$ \\
\hline MBA registrant data & September 2015 & $\begin{array}{l}\text { Number of registered medical practitioners in each field of speciality practice } \\
\text { Compulsory }\end{array}$ \\
\hline
\end{tabular}


are identified as paediatric (i.e. paediatric cardiology); however, for adult specialties, only the speciality is used (i.e. cardiology). As such, it is possible that a paediatric cardiologist may list hours worked in general paediatrics and list hours worked in 'paediatric cardiology' or 'cardiology'. Anecdotally, this is known to be confusing and potentially results in some paediatric specialists listing hours under the adult designation and then being classified by AHPRA as adult specialists when the completed forms are analysed.

The AHPRA dataset received for the present study comprised cell-by-cell numerical counts listing primary field of practice on the $x$-axis and secondary field (if applicable) on the $y$-axis. We derived two sets of numerical counts from the AHPRA dataset. First we counted medical practitioners designated as working in one of the paediatric speciality fields of interest to the study as either their primary or secondary speciality. We designated this count as 'AHPRA-1'.

To ensure we included those who may have made the error noted above in completing the survey, we further analysed the AHPRA data. For our specialties of interest, we also calculated the number of those who listed a non-paediatric (adult) field of practice in combination with either the specialist paediatrics or general paediatrics field of practice designation. We added this number to the count of AHPRA-1 and named this higher count 'AHPRA-2'. For example, the AHPRA-2 count included doctors listing hours worked in 'general paediatrics' and 'cardiology' (adult) as well as those paediatric cardiologists who completed the form correctly.

In addition, in February 2016, we conducted a search of publicly available websites in Australia to develop a fifth data source for analysis. We searched the websites of known speciality paediatric hospitals and clinics that stated they provided medical specialist care to children. A research assistant searched each website to identify the number of doctors listed as practicing at the hospital or clinic in each of the paediatric fields of practice of interest to the study. After examining the websites of known hospitals and clinics, we further conducted Internet searches for the names of various major Australian cities along with the words 'child/paediatric/hospital' and/or the names of each speciality of interest to the study (e.g. 'Melbourne child cardiology' or
'Adelaide hospital paediatric nephrology'). Some doctors were listed on more than one hospital or clinic website, but were only counted once. Registrars and those in any other training program were not included in the counts. The hospitals and clinics with doctors listed as practicing in the paediatric speciality fields of interest are listed in Table 2 .

\section{Results}

Counts of medical practitioners varied markedly for all specialties across the data sources examined. For example, counts of the number of paediatric neurologists practicing in Australia ranged between 17 and 51 doctors depending on the data source. Paediatric rheumatologist counts showed the least variability across data sources, ranging from seven to 16 doctors, a difference of approximately $120 \%$. Paediatric nephrology showed the largest variability, ranging from four to 23 doctors, a difference of over $450 \%$. Paediatric respiratory and sleep medicine showed the largest absolute range (from 15 to 62 doctors) across data sources.

The data sources were variable in their propensity to show relatively higher or lower numbers of doctors across the fields of paediatric speciality practice. For example, the AHPRA-1 dataset reported the fewest number of doctors for six of the eight fields of practice examined. Conversely, the RACP dataset reported the highest number of doctors for four of the eight fields of practice. In four fields of paediatric speciality practice, the number of medical practitioners listed on hospital websites exceeded the number listed in any other data source. Table 3 lists the counts for each field of practice for each data source.

\section{Discussion}

The main finding of the present study is that the numerical counts of paediatric medical specialists in Australia vary markedly across the data sources examined. The disparities are not consistent between the datasets. These findings may have significant implications for the way workforce data are used in healthcare planning and policy decisions in Australia. A policy decision may be made on the basis of one specific data source or another. Because all the sources used in the present study would likely be

Table 2. Hospitals and clinics identified as providing paediatric speciality care

Vic., Victoria; NSW, New South Wales, Qld, Queensland; ACT, Australian Capital Territory; Tas., Tasmania; WA, Western Australia; SA, South Australia

\begin{tabular}{llc}
\hline Speciality paediatric hospitals & $\begin{array}{l}\text { Paediatric outpatient clinics (at } \\
\text { general/other hospitals) }\end{array}$ & Paediatric speciality clinics \\
\hline $\begin{array}{l}\text { Royal Children's Hospital } \\
\text { (Melbourne, Vic.) }\end{array}$ & $\begin{array}{c}\text { Centenary Hospital for Women and } \\
\text { Children (Canberra, ACT) }\end{array}$ & Melbourne Children's Clinic (Vic.) \\
$\begin{array}{l}\text { Monash Children's Hospital } \\
\text { (Melbourne, Vic.) }\end{array}$ & $\begin{array}{l}\text { Royal Hobart Hospital (Tas.) } \\
\text { Sydney Children's Hospital } \\
\text { (Randwick, NSW) }\end{array}$ & $\begin{array}{c}\text { Melbourne Paediatric Specialists } \\
\text { (Vic.) }\end{array}$ \\
$\begin{array}{l}\text { Sydney Children's Hospital } \\
\text { (Westmead, NSW) }\end{array}$ & $\begin{array}{c}\text { Princess Margaret Hospital (Perth, } \\
\text { Whe Children's Clinic (Sydney, NSW) }\end{array}$ & $\begin{array}{c}\text { The } \\
\text { John Hunter Children's Hospital }\end{array}$ \\
$\begin{array}{l}\text { (Newcastle, NSW) } \\
\text { Lady Cilento Children's Hospital }\end{array}$ & (Adelaide, SA) & Heart Centre for Children (Westmead, \\
(Brisbane, Qld) & & NSW) \\
\hline
\end{tabular}


Table 3. Numerical counts for each of eight selected paediatric speciality fields of practice NHWD, national health workforce dataset; ${ }^{14}$ AHPRA, Australian Health Practitioner Regulation Agency; RACP, Royal Australian College of Physicians; MBA, Medical Board of Australia; ${ }^{5}$ websites, search of hospital and clinic websites. AHPRA-1 and AHPRA-2 refer to paragraphs 4 and 5 of the Methods section

\begin{tabular}{lccccccc}
\hline Field of paediatric speciality & \multicolumn{4}{c}{ No. doctors listed by data source } & \multicolumn{2}{c}{ Range in no. } \\
& NHWD & AHPRA-1 & AHPRA-2 & RACP & MBA & Websites & doctors \\
\hline Cardiology & 21 & 17 & 30 & 31 & 32 & 38 & $17-38$ \\
Endocrinology & 13 & 14 & 36 & 45 & 30 & 39 & $13-45$ \\
Gastroenterology and hepatology & 15 & 10 & 23 & 34 & 23 & 30 & $10-34$ \\
Immunology and allergy & 11 & 11 & 18 & 24 & 17 & 32 & $11-32$ \\
Nephrology & 4 & 4 & 13 & 20 & 9 & 23 & $4-23$ \\
Neurology & 22 & 17 & 44 & 46 & 32 & 51 & $17-51$ \\
Respiratory and sleep medicine & 18 & 15 & 37 & 62 & 26 & 49 & $15-62$ \\
Rheumatology & 7 & 8 & 10 & 16 & 12 & 15 & $7-16$ \\
\hline
\end{tabular}

viewed by the public and policy makers to be valid if used individually, significant misperceptions regarding the number of doctors may occur on a regular basis. For example, depending on the data source used, the current paediatric neurologist workforce in Australia comprises anywhere between 17 and 51 doctors, a range of difference of $300 \%$. The implications of a decision made using one data source may or may not be in the best interests of the public, especially as the true number is likely unknown.

It is important to note that these discrepancies identified are not due to inclusion of registrars or those in training: the data include only those who are practicing as specialists in a given field of paediatrics and/or are registered with the MBA.

The present study has identified several potential issues in the way workforce data are collected or analysed.

\section{Reliance on self-report}

Self-reported data are likely prone to more inconsistencies than objectively verified data. For example, when data are integrated into the NHWD, several medical practitioners are identified as erroneously self-reporting as specialists on the AHRPA workforce survey. These are mostly believed to be overseas specialists lacking accredited registration in Australia. ${ }^{15}$ Other mistakes or confusion may have occurred during completion of the AHPRA workforce survey. For example, specialties are identified by twodigit numeric codes that appear in a separate list on the questionnaire ${ }^{16}$ Doctors must go that location in the survey, identify the code that correlates with their speciality and then enter the number in a different place. If the survey is completed in a hurry or without attention to detail, it would be easy to mistakenly use the physician (adult) code rather than the paediatrics code for a speciality field of practice. In addition, the National Law does not require or enable doctors to identify which is their primary speciality or field of practice during the registration and renewal process. ${ }^{15}$ Primary speciality or field of practice is inferred from their response to other questions, such as the speciality field in which the doctor reports working the most hours during the preceding week. This process relies on the doctor to accurately recall their working hours during the previous week and may not reflect their regular or usual practice.

\section{Response rate}

Voluntary surveys may be subject to response bias and not represent the entire workforce being studied. For example, the response rate to the 2013 AHPRA workforce survey was $88.6 \%$ of registered medical practitioners. ${ }^{15}$ Whether non-response was more likely in some specialties than others is unknown from the available data. In addition, new doctors registering outside the normal renewal period (July-September) would not have received a survey and thus would not be included in the workforce data. ${ }^{15}$

\section{Completeness of datasets}

Some datasets may only include those who are members of specific speciality groups (e.g. speciality societies). As such, they may reflect only those who have currently paid their dues or fees to that group. Thus, these rosters may contain only a proportion of specialists and not accurately reflect the speciality as a whole.

\section{Currency of information}

Any data on the medical workforce must always be considered to be a snapshot taken at a particular point in time. ${ }^{17}$ Hospitals may not be maintaining the currency of lists of specialists on their websites. Further, if not updated regularly, practitioner status regarding clinical activity may have changed since data were reported from any source. Doctors may have periods of clinical inactivity for a variety of reasons or may go overseas for training or employment for a period of time.

\section{Accounting for actual clinical workload}

Often, workforce numerical counts and projections fail to account for a health professional's non-clinical activities ${ }^{18}$ and/or part-time status in the workforce. Other professional activities may include medical administration, research and teaching. ${ }^{2}$ Thus, when attempting to assess the actual clinical capacity of the workforce, simple numerical counts (head counts) fall short. None of the data sources examined in the present study accounted for the actual clinical working hours of a doctor, and so may give a false impression of the actual clinical availability to provide care to patients.

\section{Quality of care}

The MBA website allows one to search for a medical practitioner by name and will show the fields of practice in which the medical practitioner is currently registered. It is thereby possible to verify whether a given doctor holds registration in the fields of 
speciality practice in which they are advertised (on hospital or clinic websites). The discrepancies found between the data sources demonstrate that there are several doctors who are trained and/or self-declared to be specialists in fields of paediatric practice for which they are not registered with the MBA. From the present study, we are not able to definitively determine why this occurs or whether there are any implications for quality of care. Further studies should examine this issue in greater detail.

\section{Conclusion}

The present study identified inconsistencies across various respected paediatric specialist workforce data sources previously presumed to be authoritative. These inconsistencies point to the reality of substantial errors in these same data. To make accurate projections and plan for the future workforce, current data must be accurate and reliable. Without accurate data on which to base decision making, policy options may not be aligned with the actual needs of children with various medical requirements, in various geographic areas or the nation as a whole. As such, the number of government-supported training programs and training positions for specific paediatric subspecialties may not be congruent with the actual workforce needs of a specific state or the country.

Understanding the effective clinical workforce is crucial in addressing any workforce or waiting time issues. Any such discussion relies on accurate quantification of the current effective clinical medical workforce using numerical count data. The availability of national health workforce datasets is limited. ${ }^{9}$ As shown in the present study of eight selected fields of paediatric speciality practice, the datasets that are currently available do not appear to be consistent or reliable counts of the number of medical practitioners. Lack of accurate workforce data can affect the ability to develop rational and cogent policies to address long waiting times for paediatric speciality care in the public and private sectors of the healthcare system.

The findings of the present study have significant implications for those in government or other roles who seek to determine the current workforce providing specialist paediatric care. The lack of an adequate baseline for the workforce prevents accurate predictions of future needs to provide the best possible care of children in Australia. We posit that greater attention to the accuracy and completeness of data collected as part of the federal registration program is the best place to start.

\section{Competing interests}

None declared.

\section{References}

1 Medical Board of Australia (MBA). Registration standards for specialist registration. Melbourne: MBA; 2011. Available at: http://www.medicalboard.gov.au/documents/default.aspx?record=WD11\%2F5428\&dbid= AP\&chksum=UYvxT24Gzf73VkYq9H49aA\%3D\%3D [verified 24 September 2016].

2 Health Workforce Australia. Australia's future health workforce: doctors. Canberra: Commonwealth of Australia; 2014. Available at: http://www. health.gov.au/internet/main/publishing.nsf/Content/F3F2910B39DF55 FDCA257D94007862F9/\$File/AFHW\%20-\%20Doctors\%20report.pdf [verified 24 September 2016].
3 Health Workforce Australia. Health workforce 2025: medical specialties. Volume 3. Adelaide: Health Workforce Australia; 2012.

4 Medical Board of Australia (MBA). List of specialties, fields of specialty practice and related specialist titles. Melbourne: MBA; 2013. Available at: http://www.medicalboard.gov.au/documents/default.aspx?record= WD10\%2f106\&dbid=AP\&chksum=07LyDUkqqYa5O5LXuqbSzg $\%$ 3d\%3d [verified 24 September 2016].

5 Medical Board of Australia (MBA). Medical practitioner registrant data: September 2015. Melbourne: MBA; 2015. Available at: http://www. medicalboard.gov.au/documents/default.aspx?record=WD15\%2f18954 $\&$ dbid=AP\&chksum=8DakbZTgUf7E5sS2if7cQQ\%3d\%3d [verified 24 September 2016].

6 Department of Health. 2.3 The shortage of medical specialists and the training capacity of the health system. Canberra: Commonwealth of Australia; 2006. Available at: http://www.health.gov.au/internet/publications/publishing.nsf/Content/work-edu-spectr-mstsc-rept-toc $\sim$ workedu-spectr-mstsc-rept-2 work-edu-spectr-mstsc-rept-2-3 [verified 29 January 2016].

7 Mason J. Mason review: review of Australian Government health workforce programs. 2013. Available at: https://www.health.gov.au/ internet/main/publishing.nsf/Content/D26858F4B68834EACA257BF0001A8DDC/\$File/Review\%20of\%20Health\%20Workforce\%20programs.pdf [verified 23 September 2016].

8 Department of Health. National medical training advisory network. Canberra: Commonwealth of Australia; 2015. Available at: http:// www.health.gov.au/internet/main/publishing.nsf/Content/nmtan [verified 29 January 2016].

9 McCarty MV, Fenech BJ. Towards best practice in national health workforce planning. Med J Aust 2013; 199(5, suppl): S10-3. doi:10.5694/mja12.10309

10 Keegan B. The Cairns Post survey reveals how lack of medical specialists, crime and the economy are regions big issues. 4 January 2014. Available at: http://www.cairnspost.com.au/news/cairns/the-cairns-postsurvey-reveals-how-lack-of-medical-specialists-crime-and-the-economyare-regions-big-issues/story-fnjpusyw-1226794636561 [verified 29 January 2016].

11 Rural Doctors Association of Australia. The value of local specialist medical services to rural Australia. Manuka, ACT: Rural Doctors Association of Australia; 2009.

12 Freed GL, Nahra TA, Wheeler JR. Counting physicians: inconsistencies in a commonly used source for workforce analysis. Acad Med 2006; 81: 847-52. doi:10.1097/00001888-200609000-00017

13 Staiger DO, Auerbach DI, Buerhaus PI. Comparison of physician workforce estimates and supply projections. JAMA 2009; 302: 1674-80. doi:10.1001/jama.2009.1461

14 Health Workforce Australia. Health workforce data: national health workforce dataset. Adelaide: Health Workforce Australia; 2014. Available at: http://www.hwa.gov.au/resources/health-workforce-data [verified 22 February 2016].

15 Australian Institute of Health and Welfare (AIHW). National health workforce data set: medical practitioners 2013. Data quality statement. Canberra: AIHW; 2014.

16 Medical Board of Australia. Workforce survey form: WKSY-30. Profession: medical. 2013. Available at: www.aihw.gov.au/WorkArea/ DownloadAsset.aspx?id=60129548761 [verified 23 September 2016].

17 Stockman JA. Studies of the pediatric subspecialty workforce: overexposed and underexposed snapshots in time. Arch Pediatr Adolesc Med 2004; 158: 1185-6. doi:10.1001/archpedi.158.12.1185

18 Mayer ML, Skinner AC. Too many, too few, too concentrated? A review of the pediatric subspecialty workforce literature. Arch Pediatr Adolesc Med 2004; 158: 1158-65. doi:10.1001/archpedi.158.12.1158 